\title{
GESTIÓN DE LOS PEI EN INSTITUCIONES CON COBERTURA EDUCATIVA DE MEDELLÍN
}

RESUMEN: En el artículo se presentan los resultados del análisis de la Gestión de los Proyectos Educativos (PEI) de las Instituciones Educativas (IE) que integran el programa de cobertura educativa de la ciudad de Medellín. Se exponen los criterios que utilizan estas instituciones para autoevaluar, actualizar y revisar los PEI.

Las IE advierte en los estudiantes que participan en el programa de cobertura educativa problemáticas de agresividad, uso, consumo y transporte de sustancias psicoactivas así como bajo rendimiento académico en pruebas internas y externas, por ello demandan de la Secretaría de Educación de la ciudad acompañamiento para el manejo del riesgo, la convivencia en estas comunidades educativas así como valorarlas más allá de los resultados de las Pruebas del Saber.

Este estudio concluye que los principios y objetivos de la IE analizadas están alineados con la política y directrices que inspiran el programa de cobertura educativa de Medellín, el cual pretende formar ciudadanos con valores humanistas, socio-afectivos, democráticos, medioambientales y éticos, con competencias para el saber ser, hacer, y saber estar con otros.

Palabras Clave: PEl; educación; IE, convivencia; estudiantes; gestión educativa.

* Doctora en Estudios Científicos y Sociales- Universidad Jesuita de Guadalajara-México-Iteso. Magister en Comunicación- Universidad Iberoamericana de México-Plantel Santa Fe- UIA. Especialista en Gerencia de la Comunicación- Universidad Pontificia BolivarianaMedellín- UPB. Comunicadora Social-Periodista- Universidad de Antioquia- Colombia. Directora del Grupo de Investigación- GIC, docente e investigadora del Politécnico Colombiano Jaime Isaza Cadavid. Email: mmvalle@elpoli.edu.co

** Economista Universidad UCC, Egresado de maestría en ciencias de la administración Universidad Eafit. Consultor y Profesor en Varias Universidades de Medellin. Email: aaramires@gmail.com 


\section{MANAGEMENT OF EDUCATION PROJECTS IN EDUCATIONAL INSTITUTIONS OF MEDELLÍN}

SUMMARY: This paper analyses the results of the Institutional Education Project Management (PEI) carried out in Educational Institutions (IE) that make part of the educational coverage program in Medellín city. It presents the evaluation criteria used by these institutions to self-assess, update and review these projects.

The Educational institutions have evidenced students who are involved in the educational coverage program, present aggressiveness and consumption and traffic of psychoactive substances, as also they scored low academic performance in internal and external tests. For this reason they are claiming support from Secretaria de Educatión of the city, in issues such as risk management and educational community coexistence, due to Secretaria de Educacion often estimates these students' performance just by the results of state tests.

It is concluded that the principles and objectives of the analyzed educational institutions are aligned with the policy and guidelines that motivate the initiative of educational coverage program in Medellin city, which aims to enable citizens with humanistic, socioaffective, democratic, environmental and ethical values, to know how to be, to do, and to coexist with others.

Keywords: education project management; education; educational institutions; coexistence; students; educational management.

\section{ADMINISTRAÇÃO DOS PROJETOS EDUCACIONAIS EM INSTITUIÇÕES EDUCACIONAIS DE MEDELLÍN}

RESUMO: No artigo se apresentam os resultados da Análise da Gestão dos Projetos Educativos Institucionais (PEI) das Instituições Educativas (IE) que integram o programa de cobertura educativa da cidade de Medellín. Se espõem os critérios que estas intituições utilizam para autoevaluar, atualizar e revisar os $\mathrm{PEI}$.

As IE notam nos estudantes que participam no programa de cobertura educativa cobrir as problemáticas de agressividade, uso, consumo e transporte de substâncias psicoactivas, como também o baixo rendimento acadêmico em testes internos e externos, por isso exigem da Secretaria de Educação da cidade o acompanhamento para a gestão do risco, a convicência nestas comunidades educativas como também os avaliar-las além dos resultados dos Testes do Conhecimento.

Este estudo conclui que os princípios e objetivos das IE analisadas estão alinhados com a política e as diretrizes que inspiram o programa de cobertura educacativa de Medellín, que pretende formar cidadãos com valores humanistas, sócioafetivos, democráticos, ambientais e éticos, com competências para o saber ser, fazer, e saber estar com outros.

Palavras chaves: PEl; educação; IE, convivência; estudantes; gestão educativa. 
a cobertura educativa es un programa gubernamental que tiene por objetivo la contratación de instituciones educativas de carácter privado para que reciban a estudiantes de sectores en los que las instituciones públicas no cuentan con cupos o en los que no hay planteles. Algunos representantes de la educación sostienen que más que este tipo de programas se debe fortalecer la educación pública. En el Decreto 1851 de 2015 , se indica que la IE que integre el programa de cobertura debe cumplir con percentil de calidad superior a 20 , en una escala de 0 a 100 , lo que se define por las Pruebas Saber. Las instituciones educativas que integran este programa sostienen que se les debe considerar como oferentes más allá de sus resultados en dichas pruebas.

Según el estudio Evaluación de la Gestión Privada del Servicio Público Educativo en Medellín realizado por el Centro de Investigaciones y Consultorías de la Facultad de Ciencias Económicas de la Universidad de Antioquia, el programa de cobertura y calidad educativa de Medellín, no es un fracaso a pesar de que los estudiantes inscritos en los colegios concesionados obtienen bajos puntajes en las pruebas Icfes, los cuales llegan a ser inferiores en comparación con los de sus pares de instituciones públicas (en especial en lenguaje y matemáticas).

El estudio que se presenta a continuación concluye que los principios y objetivos de la IE analizadas están alineados con la política y directrices que inspiran el programa de cobertura educativa de Medellín, el cual pretende formar ciudadanos con valores humanistas, socio-afectivos, democráticos, medioambientales y éticos, con competencias para el saber ser, hacer, y saber estar con otros, además de educación con calidad.

\section{Resultados}

\subsection{Problemáticas de la IE}

Las principales problemáticas que presentan las Instituciones Educativas con cobertura educativa de Medellín son los mismos estudiantes favorecidos con el programa de cobertura, sus familias así como el entorno del que provienen, ver gráfica N1.
Actores de las problemáticas identificadas por las IE

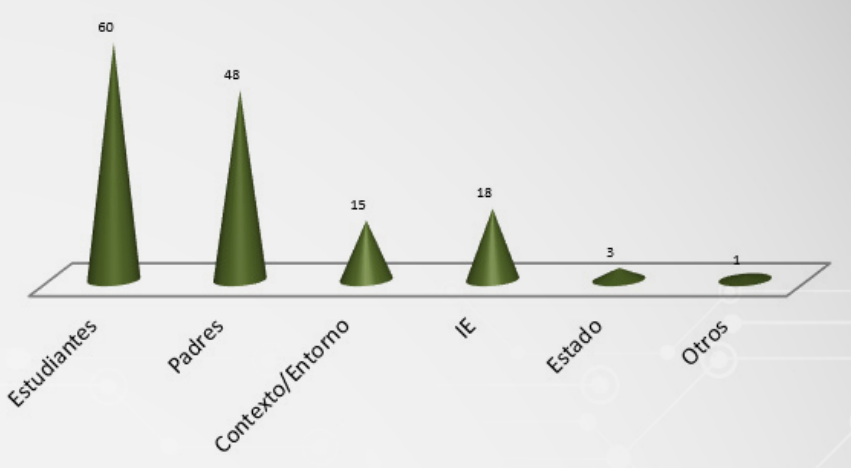

Elaboración Propia (2015)

Las problemáticas referidas a los estudiantes fueron:

...alto índice de jóvenes con dificultades
comportamentales y agresividad, alto nivel de
desinterés por el estudio, irresponsabilidad
para cumplir con los deberes escolares, bajo
rendimiento académico en pruebas internas y
externas.

Los ritmos y tiempos de aprendizaje de estos estudiantes en relación con el que presentan los estudiantes regulares es particular ya que requieren de mayor tiempo para la apropiación de los conocimientos, desarrollos de competencias y habilidades. Lo que implica a la IE invertir recursos financieros y humanos adicionales que no cubre el Programa de la cobertura educativa.

El segundo foco de problemas se encuentra en los Padres, debido al:

bajo nivel cultural y de interés por el estudio, falta de métodos, saberes e interés para ayudar a sus hijos (as) con los deberes escolares. Ausentismo en el proceso académico de sus hijos (as) debido a que permanecen fuera de casa, el bajo nivel educativo de éstos. La mayoría se dedica a las ventas ambulantes o son empleadas domésticas.

Un tercer factor problemático lo representa el contexto/ entorno:

Problemas sociales, fronteras invisibles que hacen que los estudiantes no puedan cruzar de un sitio a otro; expendio de drogas, delincuencia. Difícil situación económica. 
La falta de interés y de cultura por el manejo de la información por parte de la comunidad educativa también se constituye en problemática:

"tanto padres de familia, como profesores y comunidad en general se les dificulta hacer devolución de los desprendibles que certifican que la información - comunicados fueron leídos".

Es de advertir que aunque las IE no reportan como problemas principales a la Secretaria de Educación Municipal de Medellín ni al Ministerio de Educación, indican que:

\footnotetext{
"el sistema educativo presenta deficiencias para cubrir en su totalidad las demandas del Programa de cobertura educativa" y "aunque se tienen diversas ofertas algunos estudiantes no se ajustan a estas".
}

En relación con la Secretaria de Educación de Medellín, la IE expresan que la capacitación docente que les brinda es limitada. Señalan además que el Estado no cuenta con políticas orientadas al mejoramiento de las economías campesinas y el apoyo educativo para este sector. Convocan a que realicen encuentros para socializar y compartir experiencias exitosas del Programa de cobertura educativa y de convivencia.

\subsection{Revisión y criterios para la actualización del PEI}

El $79 \%$ de las IE consultadas indican que el PEI lo actualizan cada caño, el $11 \%$ señala que lo hace cada dos años. Un $4 \%$ lo hace cada tres años y otro tanto lo hace a más de este tiempo, un $2 \%$ no contesta la pregunta. La razón más recurrida para la actualización del PEI son las disposiciones gubernamentales.

\section{Gráfica N2.}

\section{Criterios de las IE para la actualización del PEI}

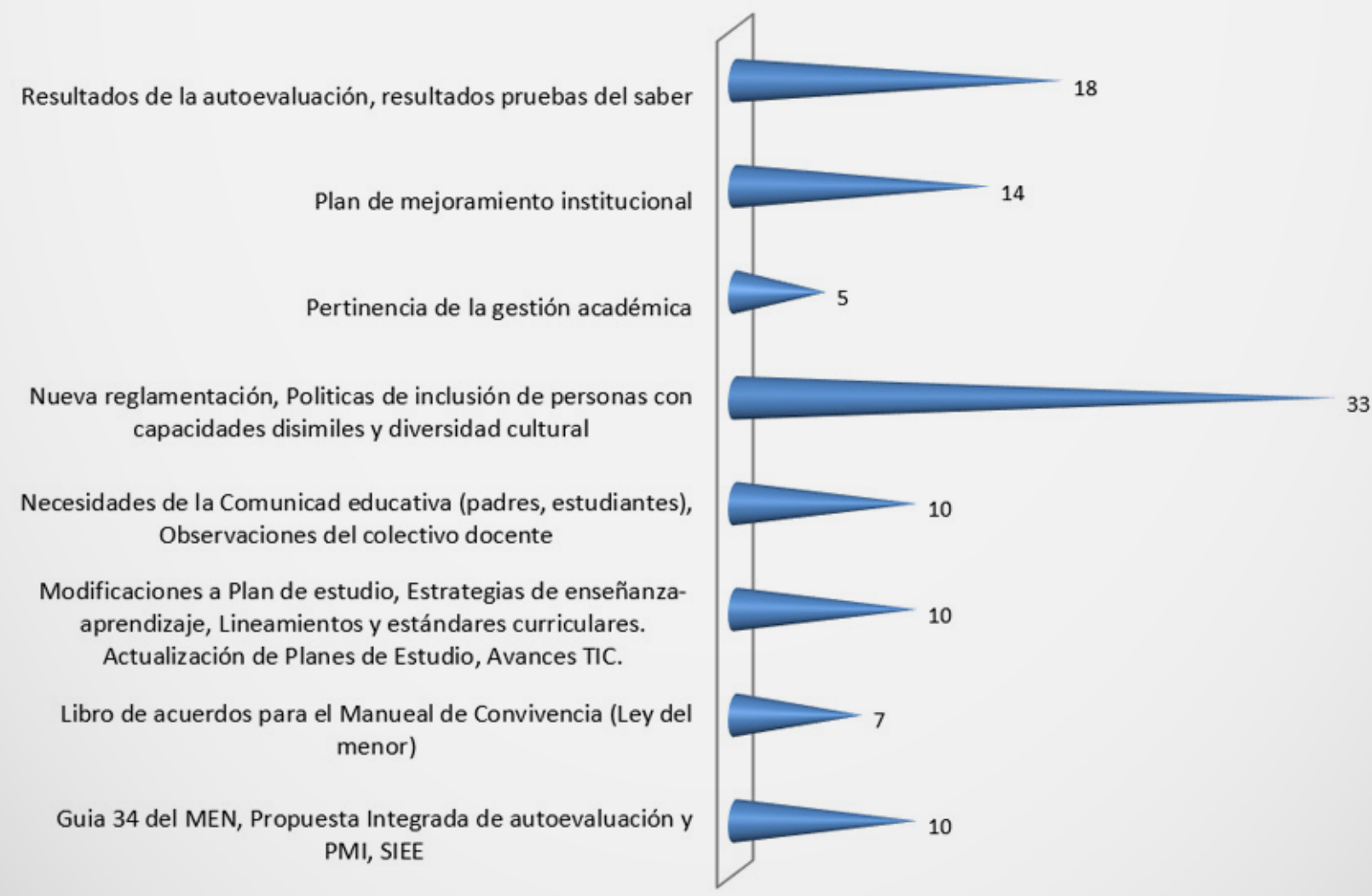


La información que se retoma para los procesos de autoevaluación proviene principalmente del Consejo académico y el directivo de la IE, también de encuestas que realizan a la comunidad institucional, el PQRSF (peticiones, quejas, reclamos, sugerencias y felicitaciones); igualmente aportan datos los procesos de gestión de la calidad, control interno y auditoria.

\section{Gráfica N3.}

\section{Formas como la IE realiza procesos de autoevaluación del PEI}

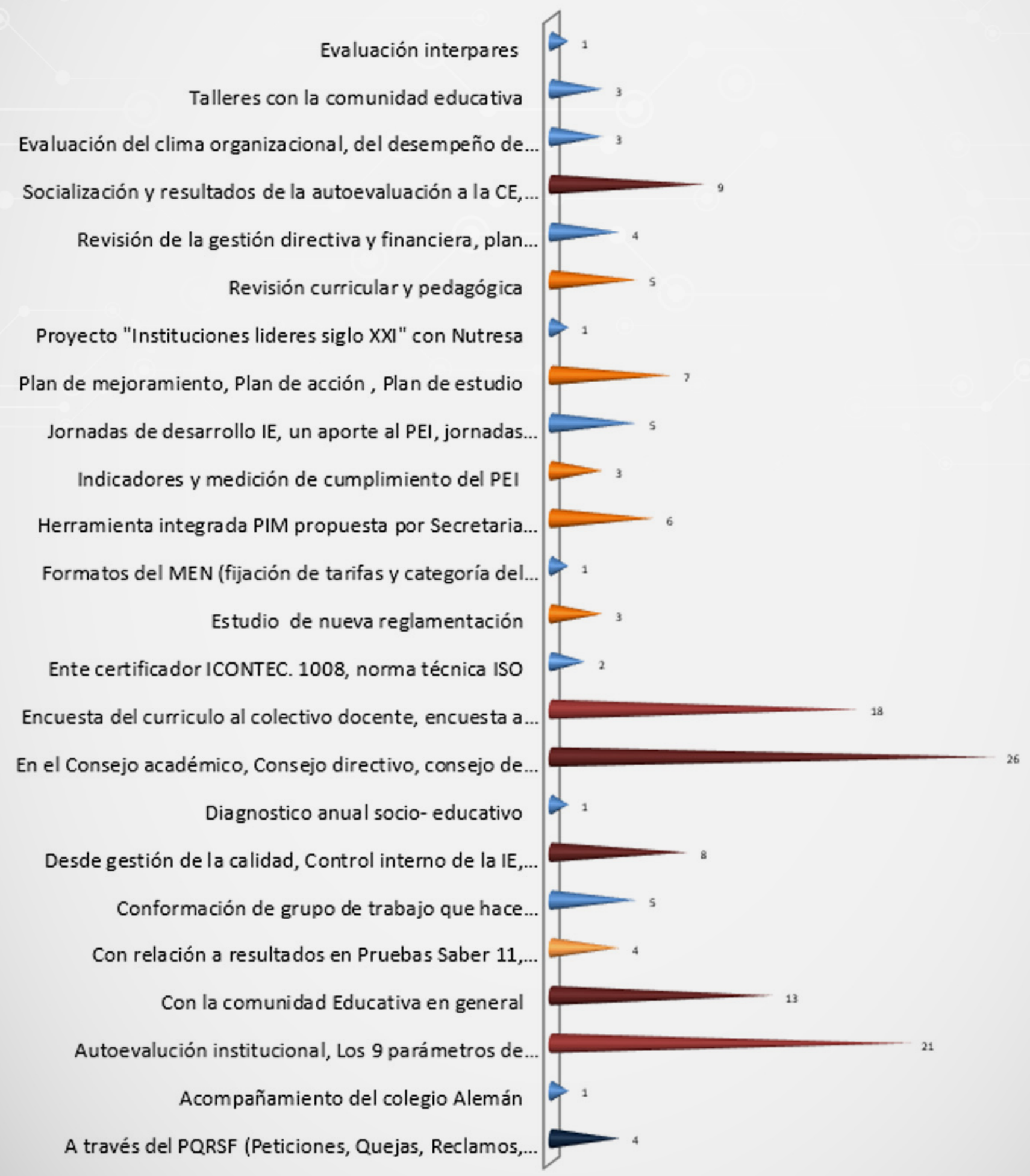


Es de advertir que solo unas pocas IE efectúan dichos procesos desde la herramienta integrada PIM propuesta por Secretaria de Educación.

\subsection{Equipos para la elaboración y/o revisión del PEI}

\section{Gráfica N4.}

Comunidad educativa que integra los equipos para Elaboración / revisión del PEI en la IE

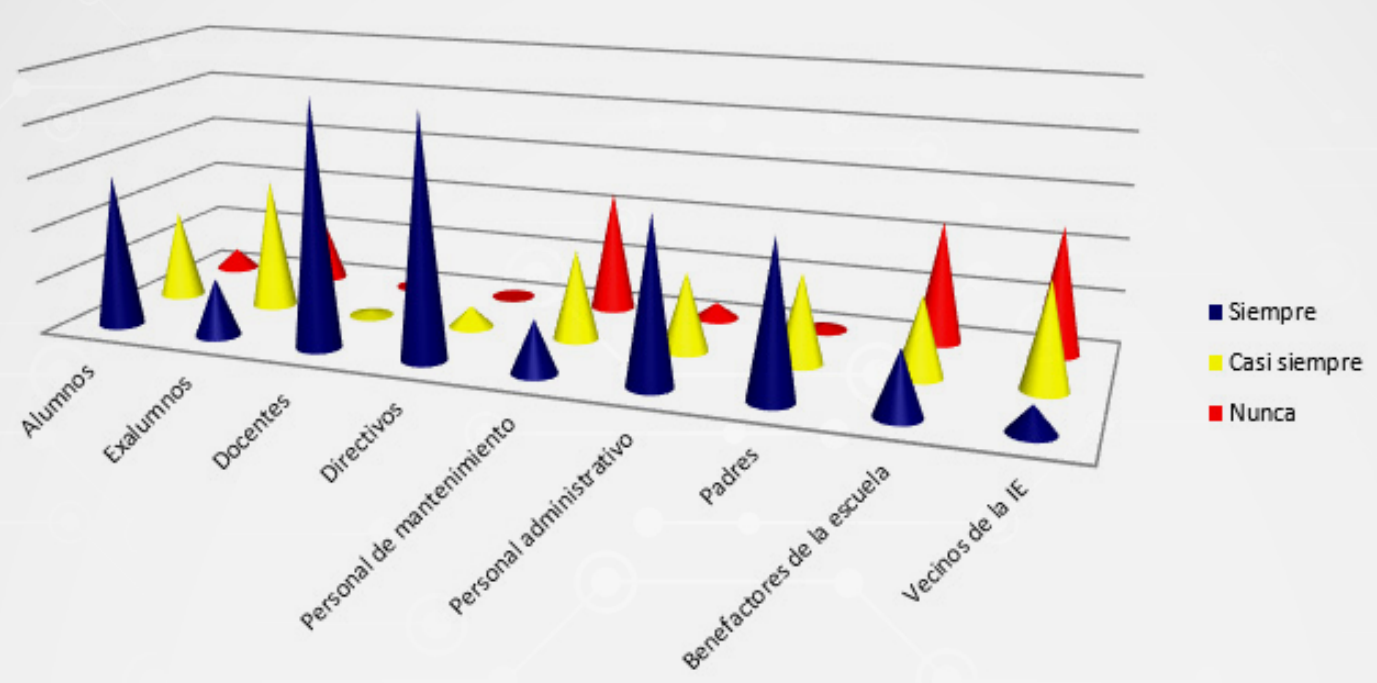

Las IE consultadas integran equipos de trabajos para la elaboración / revisión del PEI, en estos siempre participan directivos, profesores, personal administrativo, padres y alumnos. Se evidencia que los exalumnos casi nunca participan en estos procesos, salvo algunas excepciones esos equipos nunca los integran vecinos de la IE, benefactores de la escuela, el personal de mantenimiento. Lo que sin duda sería importante que lo hicieran justo para que de manera integrada se atiendan las problemáticas detectadas.

\subsection{Aspectos que se destacan de la implementación de los PEI}

En los PEI de la IE con cobertura se destacan el componente pedagógico, la gestión administrativa y los asuntos misionales. En lo misional se pretenden acciones de mejora para: apropiación de niñas, niños y jóvenes de la diversidad y la negociación no violenta de conflictos, inclusión, proyección a la comunidad, formación de líderes. Sobre sale también la filosofía institucional, la integración con la comunidad, la cultura de la legalidad, la educación inclusiva, el proyecto de convivencia, habilidades para la vida, etc.

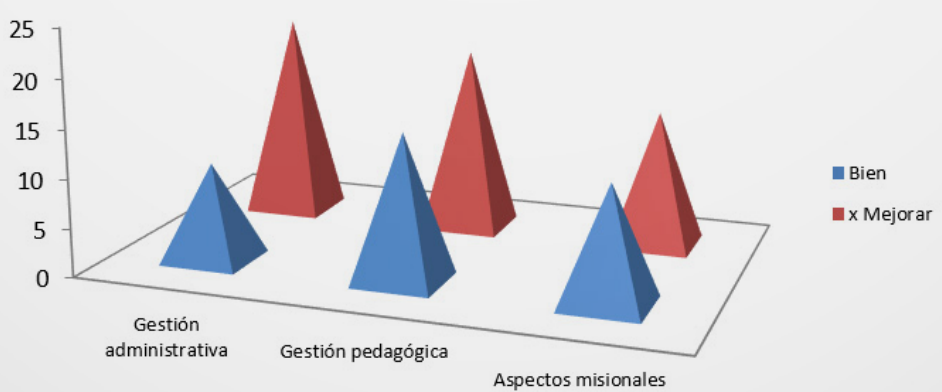


En cuanto a la gestión administrativa se resalta: "Continuidad en la Certificación ISO 9001-2008", "la evaluación Institucional", gestión directiva, organización y claridad de la I. E., actualización permanente de los componentes del PEI, seguir las normas de la Secretaria de Educación, etc. En relación a la gestión pedagógica se resalta: la flexibilidad académica, gestión pedagógica, investigación, planes de estudios, el modelo pedagógico, las didácticas curriculares, el diseño e implementación de los módulos institucionales, etc.

Como todo proceso es digno de mejorar, las IE con cobertura consideran que pueden avanzar en los tres aspectos anteriormente enunciados.

En cuanto a la gestión administrativa buscan mejorar en el desarrollo de estrategias de participación para que los padres de familia se involucren más en las actividades y procesos de la IE.

También pretenden más retroalimentación con exalumnos, gestionar espacios de ciudad para la recreación, actualización de los $\mathrm{PEI}$, mejorar las locaciones físicas, buscar recursos económicos, realizar más convenios interinstitucionales, mejorar las estrategias para la cualificación del personal docente, generar apropiación de la comunidad educativa en todas las gestiones, reflexiones con equipo en torno a los enfoques pedagógicos, generar cultura de la sistematización de procesos, evaluación más participativa del $\mathrm{PEI}$, determinar acciones para que los planes de mejoramiento se ejecuten efectivamente, mayor integridad y potencialidad de equipos de trabajo de las diferentes sedes.

En cuanto a lo pedagógico buscan avanzar en la implementación del bilingüismo, subir en el ISCE (índice sintético de calidad de la educación), mejorar los resultados en pruebas Saber- Icfes, excelencia académica. Ampliar la licencia de funcionamiento para la atención de los ciclo de media, realización de investigación escolar, ofrecer jornada complementaria, mejorar el trabajo con los estudiantes con capacidades diferentes, estrategias de evaluación del estudiante, mayor intensidad horaria para la aplicación de proyectos obligatorios, documentar a detalle la experiencia significativa, apropiación y aplicación de las estrategias didácticas y mejorar los planes de estudio.

\subsection{Sistema de matrículas en la Institución} Educativa.

Las IE con cobertura manifiestan ventajas, desventajas y aspectos por mejorar en el sistema de matrículas. Excepto $3 \mathrm{IE}$, todas tienen este proceso sistematizado. Algunas utilizan el software Globoschool [1] para instituciones educativas que se encuentra en línea y permite gestionar la información administrativa, académica y financiera. Entre los aspectos que se deben mejorar para el proceso de matrícula de los estudiantes beneficiarios del programa de cobertura son :

La realización de ficha de verificación de entrega de requisitos para aspirantes al programa, otorgar más plazo a los interesados para la entrega de certificados, motivar a los padres para que hagan la matricula en el tiempo estipulado, actualizar formato de matrículas, solicitar retiro de los estudiantes del Simat usando pre-matrícula, solicitar a la Secretaria de Educación mejorar el calendario académico, poder hacer la pre-matrícula en el mismo año lectivo y en el sistema de matrícula. Capacitación para el uso adecuado del sistema de matrículas, mejorar la comunicación entre las IE y la Secretaria de Educación. Articular las bases de datos de la IE con el SIMAT.

Consideran las IE estudiadas que el Ministerio de Educación Nacional así como la Secretaria de Educación deben conocer más la realidad rural de los estudiantes para ajustarse a ella en sus procesos educativo- administrativos y normativos.

\subsection{Deserción en la IE y principales causas}

De acuerdo con este estudio la deserción de los estudiantes beneficiarios del programa de cobertura en baja. Para el $85 \%$ de la IE con cobertura, la deserción representa entre el $5 \%$ y $10 \%$. Para el $15 \%$ de IE está entre el $16 \%$ y el $20 \%$ de sus estudiantes, sólo un $2 \%$ de las IE reportan deserción de más del $20 \%$. Entre las razones por la que los que se abandonan los estudios están:

\footnotetext{
Estudiantes embarazadas que no pueden continuar con la jornada, madres solteras que no tienen con quien dejar a los hijos(as). Otras situaciones es la falta de transporte para población extra edad y adulta, el perfil de estudiantes de los Centros de proteccióncárcel, el desplazamiento forzado.
} 
Estos estudiantes también abandonan sus estudios porque no logran desarrollar los hábitos y métodos de estudio que la IE les brinda; porque pertenecen a familias disfuncionales, por las dificultades de orden público en sus barrios, consumo de droga, por qué sus padres a pesar de la temprana edad sus hijos permiten que no asistan a los procesos académicos.

\subsection{Gestión administrativa de las IE con cobertura educativa}

\section{- Recursos con los que cuenta la IE en relación con el proceso de cobertura educativa.}

Las IE consultadas cuentan con recursos suficientes para brindar el servicio de cobertura educativa. El primero es el humano seguido del físico. El económico y el tecnológico son los que más requieren.

\section{- Calidad de la prestación del servicio de la IE en relación con la cobertura contratada}

Estas calificaron su gestión administrativa con 5 $(51 \%)$ y con $4(43 \%)$ solo un $6 \%$ la calificó con 3 . Lo que lleva a suponer que tienen una buena percepción de este proceso, así como de los servicios que brindan, los cuales califican como excelentes y buenos. Son conscientes que hay aspectos que mejorar y están dispuestas a realizar los ajustes que así se requieran.

\subsection{Análisis del Manual de convivencia, de funciones, el reglamento de docentes y el plan de riesgos físicos}

Todas las IE cuentan con Manual de convivencia, de funciones, reglamento docente y el plan de riesgos físicos. Es de señalar que 5 no cuentan con Plan de riesgos, y a 4 les falta Manual de funciones. Una IE no contesta esta pregunta y otra manifiesta que el manual de convivencia se encuentra en proceso de reestructuración.

\section{Gráfica N6.}

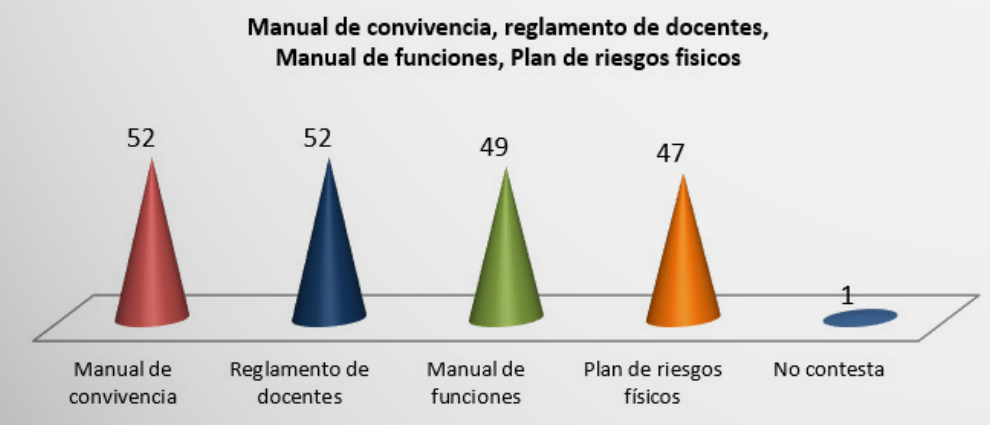

Para todas las IE estos documentos además de tener carácter obligatorio son considerados necesarios y valiosos para la función que adelantan. Algunas indican que el Manual de convivencia lo tienen adaptado y actualizado de acuerdo con la LEY 1620 del 2013 en la que se incluyen todos los conceptos y garantías para la convivencia incluyente.

\footnotetext{
Es conveniente que las IE actualicen los Manuales de Convivencia a la luz del Decreto 1075 de 2015 [2], este refiere en el Artículo 1.1.4.3. al Comité Nacional de Convivencia Escolar en el que se indica que tiene por objeto definir la operación del Sistema Nacional de Convivencia Escolar, coordinar su gestión en cada uno de sus niveles e instancias, articular sus acciones con las políticas nacionales, promover, liderar estrategias y acciones de comunicación que fomenten la reflexión sobre la convivencia escolar, la prevención, mitigación y atención del acoso escolar, la violencia escolar y la disminución del embarazo en la adolescencia. Asunto contemplado también en la Ley 1620 de 2013, artículo 8
}

Se hace necesario que dicho Manual se actualice también en términos de manejo de sustancias psicoactivas, se busquen estrategias para socializarlo tanto entre los estudiantes como entre los padres de familias. Para IE los asuntos de la convivencia escolar encuentran una guía no solo en el Manual sino y muy especialmente en el diálogo y la concertación.

Sesabequela reestructuración que se propone desde la ley 1620 tiene enfoque inclusivo, esto quiere decir, que las instituciones deben hacer acompañamiento a los grupos minoritarios (afrodecendientes, etnias, LGTBI, mujeres maltratadas, estudiantes en situación de discapacidad), lo que implica brindarles oportunidades para la expresión.

En algunas IE, en el desarrollo del Plan de riesgos físicos participan asignaturas como tecnología, artística y sociales. Otras lo trabajan en conjunto con gestión humana y la ARL. Se advierte que además de estos riesgos estas instituciones deben atender otros relacionados con el uso de las TIC, atentados con arma blanca o con armas de fuego.

Es indispensable que en las IE se implementen los simulacros de evacuación, salvamentos, así como actividades de promoción y prevención del riesgo, visibilizar este comité. En el caso de IE consideradas de alto riesgo por la población escolar que albergan o el sector en el que están ubicadas es necesario que se instruya a la comunidad educativa para atender 
este tipo de situaciones, en tal sentido la Secretaría de Educación debe acompañar procesos de valoración y atención de riesgos en las IE, y ampliar la consideración de éstos más allá de los físicos.

\section{Conclusiones}

La gestión de los Proyectos Educativos en Instituciones Educativas con cobertura educativa de Medellín es adecuada. Los criterios utilizados por estas para la actualización de sus PEI obedecen a las nuevas disposiciones gubernamentales, especialmente las referidas a la inclusión de personas con capacidades disimiles y la diversidad cultural. Los PEI también se revisan si así lo demandan sus procesos de autoevaluación, los resultados en pruebas del saber y según objetivos del Plan de mejoramiento. Se destacan en los PEI de las participantes de este estudio, los referentes pedagógicos, de gestión administrativa y los asuntos misionales.

La calidad de la IE incide positivamente en el programa de cobertura educativa por asuntos como: la transparencia en la gestión, su visión humanista y de derechos humanos, la permanente evaluación de sus procesos y comunidad educativa, el compromiso y la responsabilidad que manifiestan frente a la educación de calidad, su persistente búsqueda por mejorar la calidad académica de los estudiantes, el personal idóneo y cualificación con el que cuentan, la experiencia en la prestación del servicio educativo, la gestión de la calidad de sus procesos, el cumplimiento de la norma, la ética, el impulso al emprendimiento, su interés por la permanencia de los estudiantes en el sistema educativo, el compromiso, la formación de los profesionales en procesos de Justicia Restaurativa, el acompañamiento al estudiante en su proceso educativo, su interés por educar con responsabilidad y libertad. Sus esfuerzos por la sistematización de sus procesos, además, de su disposición a trabajar conjuntamente con el MEN, ICFES y la Secretaría de Educación para mejorar el nivel académicos de los estudiantes.

Para la revisión del PEI, las IE conforman equipos de trabajo los cuales integran especialmente los directivos, profesores y personal administrativo. Sin embargo para que estos sean más participativos es importante la vinculación de alumnos, exalumnos, padres de familia así como de vecinos de la IE, benefactores de la escuela y personal de mantenimiento.
La participación de las IE en el programa de cobertura debe contemplarse como razón para la actualización de los $\mathrm{PEI}$ ya que implica requerimientos adicionales. Para elevar el nivel académico de las IE es necesario que los PEI se actualicen según las necesidades de la comunidad educativa, especialmente de estudiantes y profesores, así como las estrategias de enseñanza - aprendizaje y las TIC.

Para la socialización de los Manuales de convivencia y de los Comités de promoción y prevención del riesgo entre estudiantes y padres de familias se requieren estrategias y acciones que generen conciencia en torno a la diversidad de género, la inclusión, convivencia pacífica, la no discriminación, etc. EI Programa mismo de cobertura educativa debe socializarse entre la comunidad educativa. En las IE deben realizarse los simulacros de evacuación, salvamentos así como brindarse capacitación e instrucciones para el manejo de actos violentos.

El Plan de riesgos debe ampliar su contenido o disponerse unos nuevos que se enfoquen a riesgos diferentes a los físicos como el uso de las TIC, de arma blanca o de fuego, consumo y transporte de sustancias psicoactivas, etc.

La deserción en las IE estudiadas representa un porcentaje bajo y obedece a situaciones particulares del estudiante tales como el embarazo, desplazamiento, etc.

Las IE participantes en el programa de cobertura de Medellín consideran que pueden mejorar en aspectos como la retroalimentación informativa con exalumnos, la gestión de espacios de ciudad para la recreación, actualización de los $\mathrm{PEI}$, mejorar las locaciones físicas, buscar recursos económicos, realizar más convenios interinstitucionales, mejorar las estrategias para la cualificación del personal docente, generar apropiación de la comunidad educativa en todas las gestiones, reflexiones con equipo en torno a los enfoques pedagógicos, generar cultura de la sistematización de procesos, evaluación más participativa del PEI, determinar acciones para que los planes de mejoramiento se ejecuten efectivamente, mayor integridad y potencialidad de equipos de trabajo de las diferentes sedes.

Es importante dimensionar que el programa de cobertura y calidad educativa pretende la generación de mayores oportunidades para el desarrollo humano obteniendo finalmente mayor equidad social. Está encaminado a fomentar el acceso y la permanencia de los estudiantes así como la excelencia en los resultados académicos. Este último aspecto hay que mejorarlo. Lo importante es que la Secretaria de Educación cuenta con unos buenos aliados para hacerlo. 


\section{Bibliografía y cibergrafía}

De Subiría Samper, Julián (2006). Los modelos pedagógicos. Hacia a una pedagogía dialogante. Magisterio Editorial.

Ministerio de Educación Nacional. Ley 115 de Febrero 8 de 1994, por la cual se expide la ley general de educación. Disponible en http://www.mineducacion. gov.co/1621/articles-85906_archivo_pdf.pdf

Ministerio de Educación Nacional. Decreto 1860 de Agosto 3 de 1994, por el cual se reglamenta parcialmente la Ley 115 de 1994, en los aspectos pedagógicos y organizativos generales. Disponible en:http://www.mineducacion.gov.co/1621/ articles-86240_archivo_pdf.pdf

Ministerio de Educación Nacional. Decreto 1075 del 26 de marzo de 2015. Disponible en: http:// redes.colombiaaprende.edu.co/ntg/men/pdf/ decreto_1075_de_2015.pdf

Tunnermann B, Carlos (2008). Módelos educativos y académicos. Hispamer. Disponible en: http://www. enriquebolanos.org/data/media/book/Modelos $\% 20$ educativos $\% 20 \mathrm{y} \% 20$ academicos.pdf

\section{Notas}

[1] http://www.globoschool.com/

[2] Ministerio de Educación Nacional. Decreto 1075 del 26 de marzo de 2015. Disponible en:

http://redes.colombiaaprende.edu.co/ntg/men/pdf/ decreto_1075_de_2015.pdf

[3]Idem

[4] SIMAT es una herramienta que permite organizar y controlar el proceso de matrícula en todas sus tapas, así como tener una fuente de información confiable y disponible para la toma de decisiones. Es un sistema de gestión de la matrícula de los estudiantes de instituciones oficiales que facilita la inscripción de alumnos nuevos, el registro y la actualización de los datos existentes del estudiante, la consulta del alumno por Institución y el traslado a otra Institución, entre otros. http://www.mineducacion.gov.co/1621/ w3-article-168883.html

\section{Para citar este artículo:}

Valle, F. Mónica y Ramírez, Álvaro (2016). Gestión de los PEl en instituciones con cobertura educativa de Medellín. Revista Luciérnaga /

Comunicación. Año 8, N16. Facultad de

Comunicación Audiovisual- Politécnico Colombiano Jaime Isaza Cadavid- PCJIC \& Facultad de

Ciencias de la Comunicación - Universidad

Autónoma de San Luis Potosí- UASLP. México. Págs.69-78.

DOI. 10.33571/revistaluciernaga.v8n16a6

Link. http://www.politecnicojic.edu.co/images/downloads/ publicaciones/revista-luciernaga/luciernaga-14/ 